\title{
Cutting Disjoint Disks by Straight Lines*
}

\author{
N. Alon, ${ }^{1}$ M. Katchalski, ${ }^{2}$ and W. R. Pulleyblank ${ }^{3}$ \\ ${ }^{1}$ Department of Mathematics, Tel Aviv University, Tel Aviv, Israel \\ ${ }^{2}$ Department of Mathematics, Technion-Israel Institute of Technology, Haifa, Israel \\ ${ }^{3}$ Department of Combinatorics and Optimization, University of Waterloo, Waterloo, Canada
}

\begin{abstract}
For $k>0$ let $f(k)$ denote the minimum integer $f$ such that, for any family of $k$ pairwise disjoint congruent disks in the plane, there is a direction $\alpha$ such that any line having direction $\alpha$ intersects at most $f$ of the disks. We determine the exact asymptotic behavior of $f(k)$ by proving that there are two positive constants $d_{1}, d_{2}$ such that $d_{1} \sqrt{k} \sqrt{\log k} \leq f(k) \leq d_{2} \sqrt{k} \sqrt{\log k}$. This result has been motivated by problems dealing with the separation of convex sets by straight lines.
\end{abstract}

\section{Introduction}

For $k>0$ let $f(k)$ denote the minimum integer $f$ such that, for any family $A$ of $k$ pairwise disjoint unit radius disks in the plane, there is a direction $\alpha$ such that any straight line having direction $\alpha$ intersects at most $f$ of the disks. In this note the following result is proved.

Theorem 1.1. There exist two positive constants $d_{1}, d_{2}$ such that

$$
d_{1} \sqrt{k} \cdot \sqrt{\log k} \leq f(k) \leq d_{2} \sqrt{k} \cdot \sqrt{\log k}
$$

for all $k>0$.

* The work of the first author was supported in part by the Allon Fellowship, by the Bat Sheva de Rothschild Foundation, by the Fund for Basic Research administered by the Israel Academy of Sciences, and by the Center for Absorbtion in Science. Work by the second author was supported by the Technion V. P.R. Fund, Grant No. 100-0679. The third author's work was supported by the Natural Sciences and Engineering Research Council, Canada, and the joint project "Combinatorial Optimization" of the Natural Science and Engineering Research Council (NSERC), Canada, and the German Research Association (Deutsche Forschungsgemeinschaft, SFB 303). 
The proof of the upper bound is by a counting argument. The proof of the lower bound is more complicated. The main tool here is a well-known construction due to Besicovitch [1], which is implicit in his brilliant solution to the Kakeya problem. Besicovitch used this construction to show that there are plane figures of arbitrary small area which permit a unit segment to change its direction by $360^{\circ}$ while moving continuously within them.

Theorem 1.1 has been motivated by a result of Tvergerg [2], which shows that for every positive integer $n$ there exists a number $k=k(n)$ with the property that if $C_{1}, C_{2}, \ldots, C_{k}$ are nonempty convex sets in the plane with pairwise disjoint relative interiors, then there is a closed halfplane which contains $n$ of the sets while the closure of its complement contains at least one of the remaining $k-n$ sets. He also describes an example, attributed to K. P. Villanger, which shows that we cannot require two sets to be contained in the closure of the complement. This example consists of an arbitrary number of noncollinear line segments such that the convex hull of any pair contains the point $(1,1)$ in its interior. Consequently, there can exist no halfplane such that two segments are contained in the halfplane and two are contained in the closure of its complement.

However, if we require all the convex sets to be congruent disks, then the situation is entirely different and there exists a line with approximately half the disks on each side. Indeed, by Theorem 1.1 there is a direction $\alpha$ such that any line in this direction intersects at most $d_{2} \sqrt{k} \sqrt{\log k}$ disks. Choose a line having direction $\alpha$ such that, for either side of the line, at least $k / 2$ disks lie on that side or on the line. Since at most $d_{2} \sqrt{k} \sqrt{\log k}$ disks lie on the line, we conclude that the following holds.

Theorem 1.2. There exists a constant $c>0$ such that if $A$ is any family of $k$ congruent nonoverlapping disks in the plane, then there is a straight line with at least $k / 2-c \sqrt{k} \sqrt{\log k}$ disks completely contained on each side of $i t$.

\section{The Upper Bound of Theorem 1.1}

Let $A$ be a set of $k$ pairwise disjoint unit radius disks in the plane. Put $r=$ $\lfloor\sqrt{k} / \sqrt{\log k}\rfloor$. For $0 \leq i<r / 2$, let $l_{t}$ be a line that forms an angle $(i / r) \pi$ with the positive part of the $x$ axis, such that among all lines in this direction, $l_{t}$ intersects the maximum number of disks of $A$. To establish the upper bound of Theorem 1.1 it is enough to show that one of these $l$ 's intersects at most $O(\sqrt{k} \cdot \sqrt{\log k})$ members of $A$. An easy geometric argument shows that, for $i \neq j$, the number of disks that intersect both $l_{i}$ and $l_{j}$ is at most $O(r /|i-j|)$. Indeed, since $\sin \theta \geq 2 \theta / \pi$ for all $\theta$ satisfying $0 \leq \theta \leq \pi / 2$, we can easily check that all of these disks lie completely within an appropriate $4 \times(2+2 r /|i-j|)$ rectangle. Hence their total area (which is their number multiplied by $\pi$ ) is $O(r /|i-j|)$, as needed. It follows that for each fixed $i, 0<i \leq r / 2$, the number of disks that intersect $l_{i}$ and at least one of the other $l_{j}$ 's is at most $O\left(r \cdot 2 \sum_{j=1}^{r / 2} 1 / j\right)=O(\sqrt{k} \cdot \sqrt{\log k})$. Since the total number of disks is $k$ there exists an $i_{0}, 0 \leq i_{0}<r / 2$, such that the number of disks 
that intersect $l_{k,}$ and not any other $l$, is at most $k /(r / 2)=O(\sqrt{k} \cdot \sqrt{\log k})$. Thus the total number of disks that intersect $l_{4,}$ is $O(\sqrt{k} \cdot \sqrt{\log k})$, completing the proof.

\section{The Lower Bound of Theorem 1.1}

The proof relies on several lemmas. The first one is a modified version, due to Perron and Schoenberg, of a theorem of Besicovitch. A detailed proof of this lemma appears in [1].

Lemma 3.1. Let $p \geq 2$ be an integer and let $\triangle=O A B$ be an isosceles right triangle with hypotenuse $A B$ and area $S$. Divide the base $A B$ into $m=2^{p-2}$ equal parts by the points $A=A_{0}, A_{1}, A_{2}, \ldots, A_{m}=B$ and let $\Delta_{i}$ be the triangle $O A_{i-1} A_{i}$ $(1 \leq i \leq m)$. Then it is possible to translate each $\Delta_{i}$ along the base $A B$ so that the area covered by all these translated triangles is at most $2 S / p$.

For $n>5$ and for a $5 \times n$ rectangle $R$ in the plane, we say that the direction of $R$ is $\alpha$ if its long edge has direction $\alpha$.

Lemma 3.2. For every $n>5$ there exists a set $A_{n}$ in the plane with area $O\left(n^{2} / \log n\right)$ that contains a $5 \times n$ rectangle in every direction.

Proof. Clearly, it is enough to construct a set $B_{n}$ of area $O\left(n^{2} / \log n\right)$ containing a $5 \times n$ rectangle in every direction $\alpha, 0 \leq \alpha \leq \pi / 2$, since then $A_{n}$ can be obtained from the union of two such sets. Let $\triangle=O A B$ be an isosceles right triangle with hypotenuse $A B$ of length $2^{p-2} \times 10$, where $p-2=\left[\log _{2} n\right]$. Assume the angle $\varangle A O B$ of $\triangle$ includes all directions $\alpha, 0 \leq \alpha \leq \pi / 2$. Split the base $A B$ of $T$ into $2^{p-2}$ equal parts by the points $A=A_{0}, A_{1}, A_{2}, \ldots, A_{n}=B$ and let $\Delta_{i}$ be the triangle $O A_{t-1} A_{i}, 1 \leq i \leq 2^{p-2}$. We can easily check that for each $\alpha, 0 \leq \alpha \leq \pi / 2$, at least one of the $\Delta_{i}$ 's contains a $5 \times n$ rectangle in direction $\alpha$. By Lemma 3.1 it is possible to translate the $\Delta_{i}$ 's along the line $A B$ to obtain a set $B_{n}$ of area $O($ area $(\Delta) / \log n)=O\left(n^{2} / \log n\right)$. This set clearly contains a $5 \times n$ rectangle in each direction $\alpha, 0 \leq \alpha \leq \pi / 2$, as needed.

Remark 3.1. Using the argument of Section 2, it is not difficult to show that the area of any set that contains a $5 \times n$ rectangle in every direction is at least $\Omega\left(n^{2} / \log n\right)$. We omit the details.

Lemma 3.3. Let $R$ be a $5 \times n$ rectangle in direction $\alpha$ in the plane and let $A$ be a family of $\Omega(n)$ pairwise disjoint unit disks whose centers lie in $A$. Then there is a straight line in direction $\alpha$ that intersects $\Omega(n)$ disks.

Proof. Let $L$ be a set of five lines, each parallel to the long edge of $R$, that cut $R$ into six equal parts. Obviously each disk in $A$ intersects at least one member of $L$, and the desired result follows. 
The lower bound of Theorem 1.1 follows from the next lemma by substituting $k=O\left(n^{2} / \log n\right)$.

Lemma 3.4. There exists a set $A$ of at most $O\left(n^{2} / \log n\right)$ pairwise disjoint unit disks in the plane, such that for every direction $\alpha$ there is a line having direction $\alpha$ that intersects at least $\Omega(n)$ members of $A$.

Proof. By Lemma 3.2 there exists a set $A_{n}$ of area $O\left(n^{2} / \log n\right)$ that contains a $5 \times n$ rectangle in every direction. Let $C$ be a maximal (with respect to containment) subset of $A_{n}$ that satisfies the following two conditions:

(i) The distance between any two distinct points in $C$ is greater than 2 .

(ii) The distance between any point of $C$ and any point outside $A_{n}$ is greater than 1 .

Let $A$ be the family of all unit disks whose centers lie in $C$.

Claim 1. $|C| \leq \operatorname{area}\left(A_{n}\right) / \pi \leq O\left(n^{2} / \log n\right)$.

Proof. The members of $A$ are pairwise disjoint, each has area $\pi$, and all of them lie inside $\boldsymbol{A}_{n}$.

Claim 2. If $R$ is any $5 \times n$ rectangle contained in $A_{n}$, then there are at least $(n-4) / 4=\Omega(n)$ points of $C$ inside $R$.

Proof. Consider the family of $|C|$ disks, each of radius 2, with centers in $C$. By the maximality of $C$, each point of $A_{n}$ whose distance from the complement of $A_{n}$ is bigger than 1 lies in at least one of these disks (since, otherwise, this point can be added to $C$ ). In particular, the set $\bar{R}$ of all points of $R$ whose distance from the boundary of $R$ is at least 2 lie in these disks, and points of $\bar{R}$ can lie only in disks whose centers are inside $R$. The total area of $\bar{R}$ is $n-4$, and since each disk of radius 2 cannot cover more than area 4 of $\bar{R}$, the claim follows.

We now show that the set $A$ satisfies the assertions of Lemma 3.4. By Claim 1 the cardinality of $A$ is at most $O\left(n^{2} / \log n\right)$, and by the definition of $C$ the disks in $A$ are pairwise disjoint. Let $\alpha$ be an arbitrary direction. By construction, $A_{n}$ contains a $5 \times n$ rectangle $R$ having direction $\alpha$. By Claim 2 there are $\Omega(n)$ points of $C$ inside $R$ and hence, by Lemma 3.3, there is a line in direction $\alpha$ that intersects $\Omega(n)$ disks. This completes the proof of the lemma, and the lower bound of Theorem 1.1 follows.

\section{Concluding Remarks}

The proof of Theorem 1.1 can be easily generalized to arbitrary families of planar pairwise disjoint convex sets, each of diameter at most 1 and area at least $\gamma$ for 
any $\gamma>0$. Thus, for any $\gamma>0$ there is a positive constant $c(\gamma)$ such that for every family $A$ of $k$ pairwise disjoint convex sets, each of diameter at most 1 and area at least $\gamma$, there is a direction $\alpha$ so that each line in direction $\alpha$ intersects at most $c(\gamma) \cdot \sqrt{k} \sqrt{\log k}$ members of $A$. Similarly, for any $\gamma>0$ there is a positive constant $d(\gamma)$ such that for any sequence $\left(B_{1}, B_{2}, \ldots, B_{k}\right)$ on convex sets, each of diameter at most 1 and area at least $\gamma$, there is a family $A=\left\{A_{1}, \ldots, A_{k}\right\}$ of nonoverlapping convex sets in the plane, where $A_{i}$ is a translate of $B_{i}$ for $1 \leq i \leq k$, and for each direction $\alpha$ there is a straight line having this direction that intersects at least $d(\gamma) \sqrt{k} \cdot \sqrt{\log k}$ members of $A$. These two results follow from the proofs above by observing that for any $\gamma>0$ there is a positive constant $r(\gamma)$ such that each convex set of diameter at most 1 and area at least $\gamma$ contains a disk of radius $r(\gamma)$.

Using these results it is possible to generalize Theorem 1.2 and obtain:

Theorem 4.1. For any $\alpha>0$, there exists a constant $c=c(\alpha)>0$ such that if $A$ is a family of $k$ nonoverlapping convex sets, each of diameter at most 1 and area at least $\alpha$, then there exists a straight line with $k / 2-c \sqrt{k} \sqrt{\log k}$ sets completely contained on each side of it.

We conclude this paper with an open problem related to Tverberg's result. Given a family of $n$ disjoint segments, it is easy to verify that there exists a line which separates one of the segments from $(n-1) / 4$ others. Can the $(n-1) / 4$ be improved? We conjecture that $(n-1) / 4$ can be replaced by a number close to $n / 2$. (It cannot be replaced by a number larger than $\lceil n / 2\rceil+1$.)

\section{References}

1. A. S. Besicovitch, The Kakeya problem, Amer. Math, Monthly 70 (1963), 697-706.

2. H. Tverberg, A separation property of plane convex sets, Math. Scand. 45 (1979), 255-260.

Received September 2, 1987. 